\title{
Patients with osteosarcoma and soft tissue sarcoma might become "cancer refugees" in some Japanese regional cities
}

This article was published in the following Dove Press journal: Cancer Management and Research

\author{
Hiromichi Yamane' \\ Toshiyuki Kunisada² \\ Toshifumi Ozaki² \\ Nobuaki Ochi' \\ Yoshihiro Honda' \\ Yasunari Nagasaki' \\ Nozomu Nakagawa' \\ Tomoko Yamagishi' \\ Hidekazu Nakanishi' \\ Nagio Takigawa' \\ 'Department of General Internal \\ Medicine 4, Kawasaki Medical School, \\ Okayama, Japan; ${ }^{2}$ Department of \\ Orthopedics, Okayama University \\ Graduate School of Medicine, \\ Dentistry, and Pharmaceutical \\ Sciences, Okayama, Japan
}

Correspondence: Hiromichi Yamane Kawasaki Medical School, 2-6-I,

Nakasange, Kita-ku,

Okayama 700-8505, Japan

$\mathrm{Tel}+8 \mathrm{I} 8622521 \mathrm{II}$

Fax +81862328343

Email hiromichi.ya@gmail.com
Purpose: "Rare cancer" is defined as malignancy with a disease prevalence (age-adjusted incidence rate) of less than six per 100,000 population. Proper treatments which these patients need cannot always be performed unless they find dedicated facilities. Patients tend to be desperate, searching for advice and care. Thus, they are called "cancer refugees". Osteosarcoma and soft tissue sarcoma (OS/STS) are representative rare cancers in Japan. We conducted a retrospective analysis of patients with OS/STS to improve the current treatment modalities in a Japanese regional city. Patients and methods: Twenty-one patients with OS/STS who were hospitalized to receive standard chemotherapy or palliative treatment were enrolled between October 2011 and January 2017. Patients with non-Hodgkin's lymphoma (NHL) and advanced cancer who were treated in the palliative care unit (PCU) of the Kawasaki Medical School General Medical Center were recruited as the control groups. We analyzed the difference in residential area between patients with OS/STS and the control groups.

Results: Approximately one-third of patients with OS/STS were referred from hospitals outside of Okayama prefecture. The ratio of patients with OS/STS referred from Okayama city and/or the same medical administration area of Okayama prefecture was lower than that of patients with NHL and advanced cancer who were treated in the PCU.

Conclusion: Because the medical environment of patients with OS/STS in Japanese local cities has not been consolidated, completing medical care within the patient's own medical administration area is difficult. Thus, some patients with OS/STS may become "cancer refugees" who are unable to receive standard therapy near their residence.

Keywords: osteosarcoma, soft tissue sarcoma, rare cancer, cancer refugee

\section{Introduction}

In 2015, the Japanese Ministry of Health, Labor, and Welfare defined "rare cancer" as disease prevalence (age-adjusted incidence rate) of less than six per 100,000 population based on the RARECARE definition. ${ }^{1}$ By far, multifarious problems concerning the treatment of rare cancer, except its rarity, have been discussed. ${ }^{2,3}$ Although professional medical care programs are necessary to treat patients with rare cancer, medical experts for pathological diagnoses, clinical oncology, and surgery are lacking, and only few cancer treatment facilities are available apart from the foundation hospitals in large Japanese cities. Presumably, developing a systematic human resource and initiating basic and/or clinical research for the establishment of standard medical care in this field is difficult. ${ }^{3}$ Although a medical system to provide adequate treatment for patients with rare cancer is necessary, appropriate political measures are yet 
to be adequately provided, particularly within Japan's local cities. $^{2}$ Such problems affect the treatment of patients with rare orthopedic cancers, such as osteosarcoma and soft tissue sarcoma (OS/STS). ${ }^{4}$ Moreover, only few specialized facilities for OS/STS are available, and they are located only in metropolitan Japanese areas.

Here, we performed a retrospective analysis to clarify the trends in residence area of patients with OS/STS to overcome the current sparsity of treatment facilities and modalities for these rare tumors in our medical community.

\section{Patients and methods}

\section{Study population}

Twenty-one patients with OS/STS who were hospitalized to receive standard chemotherapy $(n=19)$ or palliative treatment $(n=2)$ in the Department of General Internal Medicine 4, Kawasaki Medical School General Medical Center, were recruited for analysis between October 2011 and January 2017. OS/STS was diagnosed pathologically based on the World Health Organization classification of soft tissue and bone tumors. ${ }^{5}$ Geographical data on the patients' residence were collected using medical records. Patients with non-Hodgkin's lymphoma (NHL) $(\mathrm{n}=37)$ and those with advanced malignant tumor $(\mathrm{n}=36)$ who were admitted to our palliative care unit (PCU) were recruited as a control group, because we expected that most of them might reside in Okayama city or neighborhood, and data on their residence were collected between October 2011 and January 2017 and between December 2016 and May 2017, respectively. Because the prevalence of NHL in Japan in 2005 was 10.6 per 100,000 population, ${ }^{4}$ this disease was considered suitable for a control group that is not a "rare cancer", but a relatively "low prevalence tumor". Patients with advanced cancer who need to be treated in the PCU tend to wish for treatment near their residence. ${ }^{6}$ Because the prevalence of those who wish to be treated near their residence was approximately equal to the general prevalence of patients with cancer $(337.5$ per 100,000 population in Japan), we selected patients with advanced cancer as a control group. Data on the residence of patients with OS/STS were collected and analyzed, and the patients were divided into the following three groups: group A comprised patients from Okayama city; group B comprised patients from outside of Okayama city, but from the same medical administration area in Okayama prefecture; and group C comprised patients from outside of Okayama prefecture. Each ratio of the patient's residence was compared with that of the control groups. This study was approved by the Kawasaki Medical University Ethics Committee (no. 2682) and conformed to the Declaration of Helsinki (1975). Written informed consent was provided by all participants before initiating treatment. None of the patients who provided consent were eliminated from this study.

\section{Statistical analysis}

The chi-squared test for goodness of fit or Fisher's exact probability test was used to test positive quantitative differences between the groups. All $P$-values corresponded to two-sided tests, and significance was set at $P<0.05$.

\section{Results \\ Demographic characteristics of the study population}

The characteristics of the patients with OS/STS in this study are outlined in Figure 1. The group comprised 21 patients
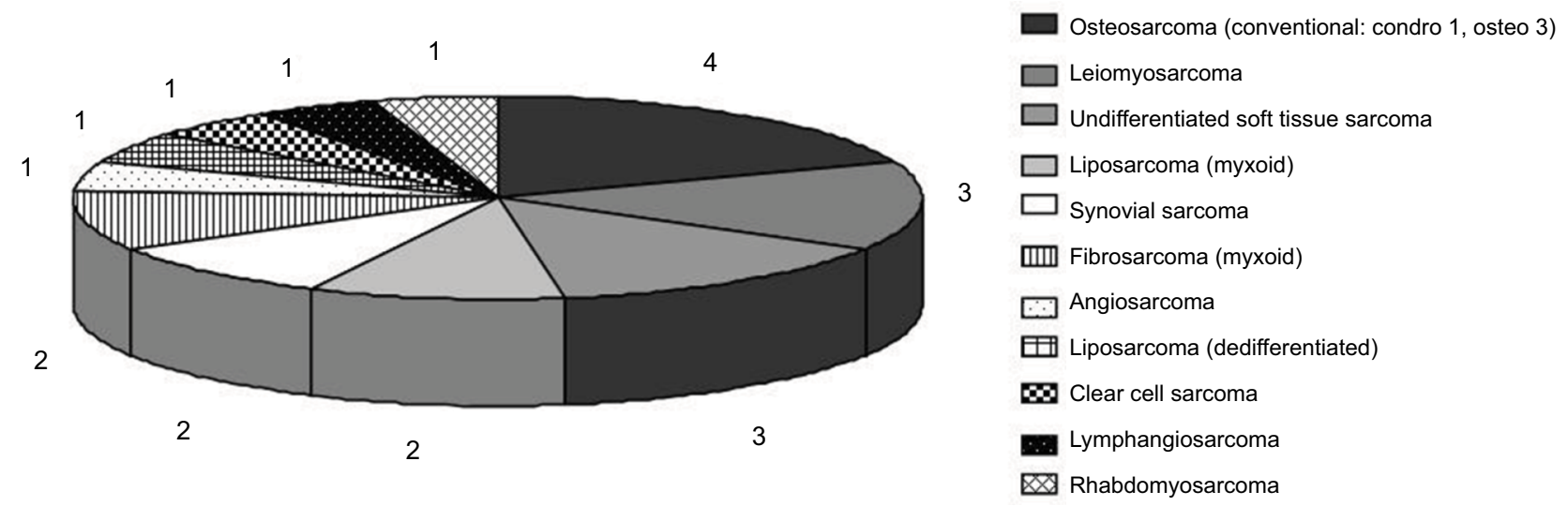

Figure I Characteristics of the patients with osteosarcoma (OS) and soft tissue sarcoma (STS)

Notes: Four patients with OS and 17 patients with STS were included in this study. Among the four patients with OS, three had osteoblastic (osteo) and one had chondroblastic (chondro) OS. All cases were classified as conventional OS. Meanwhile, liposarcoma, undifferentiated sarcoma, leiomyosarcoma, synovial sarcoma, and fibrosarcoma were the common histological diagnosis among the 17 patients with STS, comprising over $70 \%$ of diagnosis. Numbers I-4 indicate number of patients. 
(13 men and eight women) aged 25-75 years (mean \pm SD: $52.0 \pm 16.3$ years). Four patients had OS, while 17 patients had STS. Among the four patients with OS, three patients had osteoblastic OS, and one had chondroblastic OS. All cases of OS were conventional OS. The common histological diagnosis among the 17 patients with STS was liposarcoma $(\mathrm{n}=3)$, undifferentiated sarcoma $(\mathrm{n}=3)$, leiomyosarcoma $(\mathrm{n}=3)$, synovial sarcoma $(\mathrm{n}=2)$, and fibrosarcoma $(\mathrm{n}=2)$, which comprised over $70 \%$ of diagnoses (Figure 1).

The characteristics of patients treated in the PCU of our institute $(n=36)$ are shown in Figure 2. Data from these patients were used as control. The most common malignancy among these patients was lung cancer. Colorectal cancer, lung cancer, and gastric cancer, which are among the "five major cancers in Japan" along with hepatic and breast cancers, accounted for the majority of diagnosed cancers in this group at over $60 \%$ (Figure 2).

\section{Geographical data of patients' residence}

The geographical illustration of the patients' residence is shown in Figure 3. Only 33\% of the patients with OS/STS (7/21) lived within Okayama city. Furthermore, $52.4 \%$ of patients with OS/STS (11/21) did not live in the medical administration area (i.e., south-east area of Okayama prefecture) where our facilities are located. On the other hand, $28.6 \%$ of patients with OS/STS (6/21) were referred from hospitals outside of Okayama prefecture (Figure 3A). Unlike these results, substantially different data were obtained from the analysis of the control groups. The geographical data of the residence of the patients with NHL and those treated in the PCU of our institute are shown in Figure 3B and C. Twenty-two of 37 patients with NHL (59.4\%) and 31 of 36 patients treated in PCU (86.1\%) lived in Okayama city. Moreover, 29 of 37 patients with NHL (78.4\%) and 34 of 36 patients treated in PCU (94.4\%) lived in our medical administration area. Only eight patients with NHL (21.6\%) and two patients treated in the PCU (5.56\%) lived in another medical administration area of Okayama prefecture. In contrast to patients with OS/STS, no patients were referred from hospitals outside Okayama prefecture in the control groups (Figure 3B and C).

Statistical analysis of these geographical data is summarized in Figure 4. The ratio of the patients with OS/STS from Okayama city $(33.3 \%$; $95 \%$ CI: $14.6 \%-57.0 \%)$ was significantly lower than those who were treated in the PCU (86.1\%; 95\% CI: 70.5\%-93.5\%); $P=0.0000455)$. Although the ratio of patients with OS/STS and NHL from Okayama city was not significantly different $(P=0.0558)$, the ratio of patients with OS/STS from Okayama city tended to be lower (Figure 4A). The ratio of patients with OS/STS from the same medical administration area of Okayama prefecture (47.6\%; 95\% CI: $25.7 \%-70.2 \%$ ) was significantly lower than those who were treated in the PCU $(94.4 \%$; $95 \%$ CI: $81.3 \%-99.3 \% ; P=0.0000482)$ and those with NHL $(78.3 \%$;
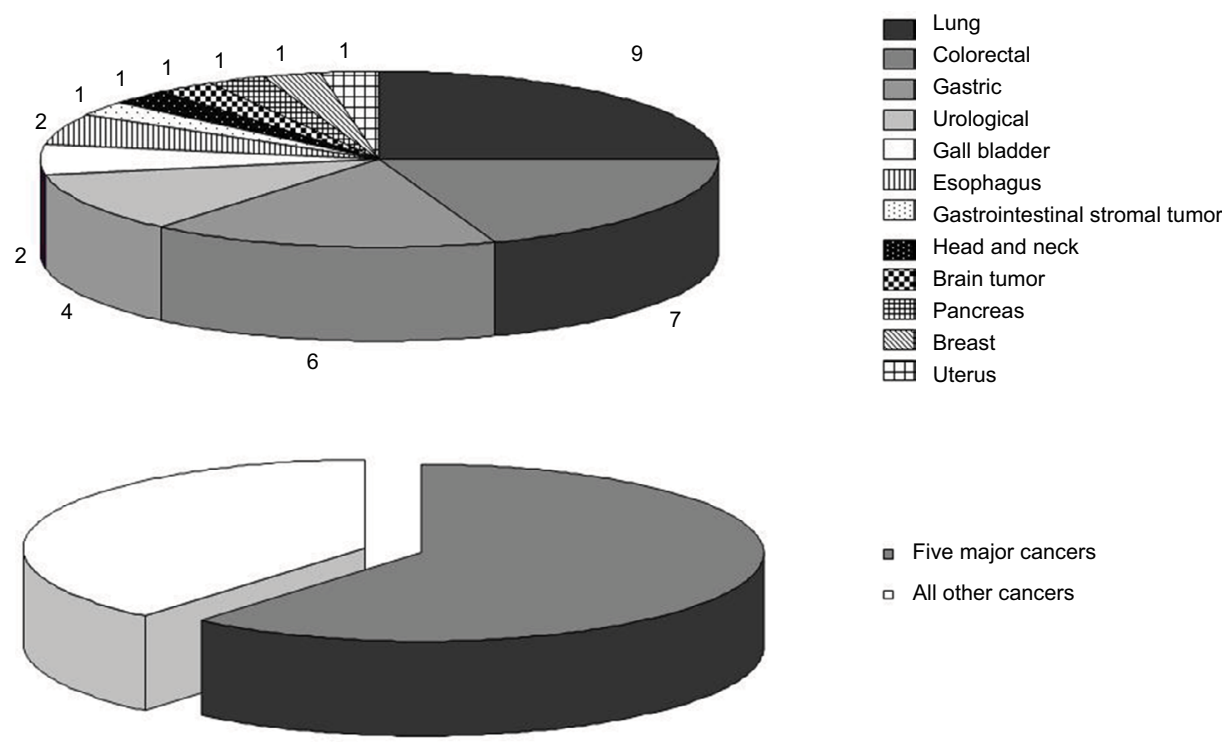

口 Five major cancers
口 All other cancers

Figure 2 Characteristics of the patients treated in our palliative care unit (PCU).

Notes: Lung cancer was the most frequent malignancy among the patients treated in the PCU. Lung cancer, colorectal cancer, and gastric cancer, which are among the "five major cancers in Japan", accounted for the majority of diagnoses at over $60 \%$. Numbers I-9 indicate number of patients. 

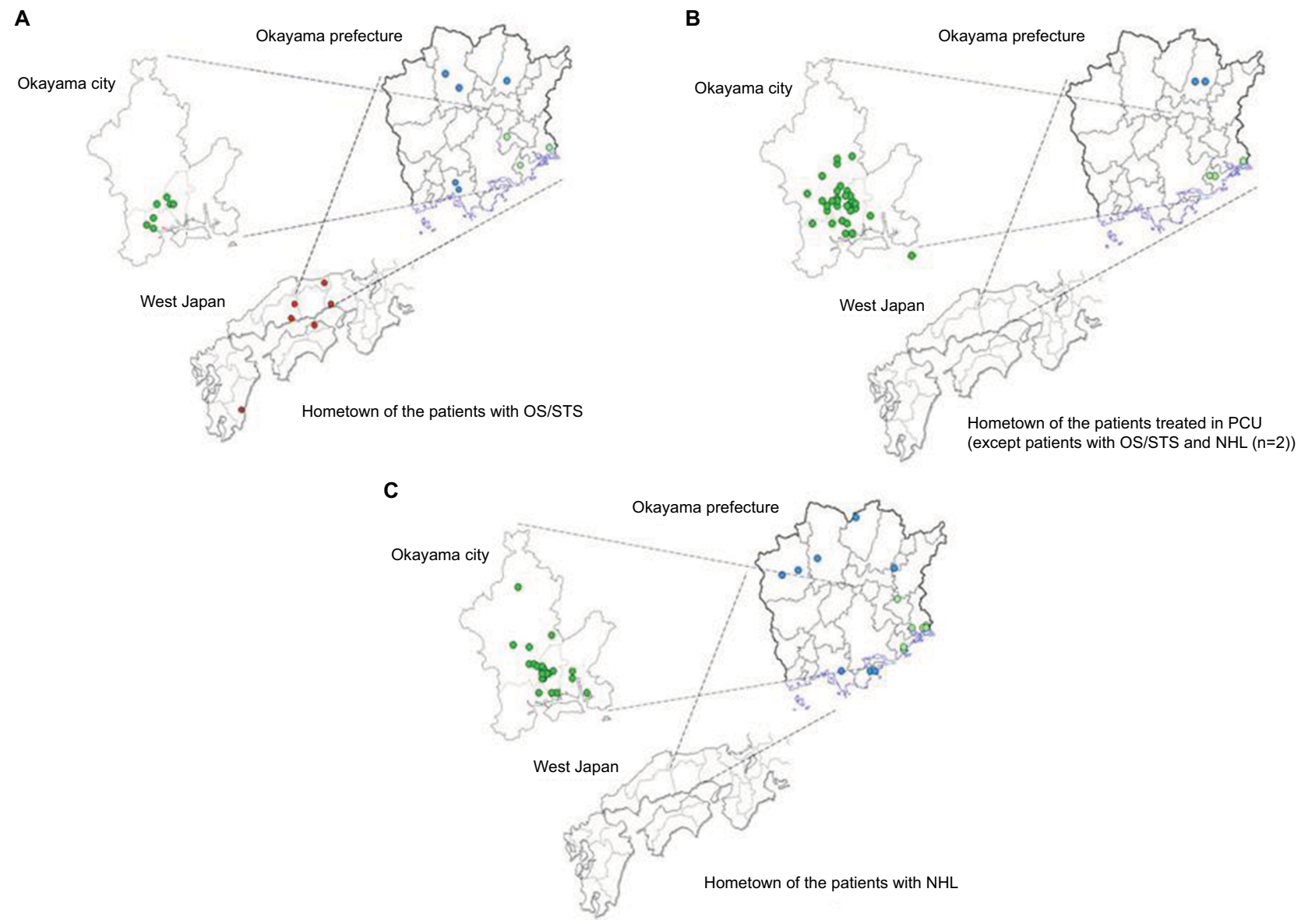

Figure 3 Number of patients referred from hospitals outside and within Okayama prefecture.

Notes: The green circles indicate the areas of residence of patients who were referred from the same medical administration areas in which our facilities are located. The blue circles indicate the areas of residence of patients who were referred from other medical administration areas in the Okayama prefecture. The red circles indicate the areas of residence of patients who were referred from other prefectures. Approximately half of our patients with OS/STS (I I/2I) did not live in our medical administration areas (A). On the other hand, all patients with NHL or patients treated in our PCU unit were referred from hospitals in Okayama prefecture (B, C).

Abbreviations: OS/STS, osteosarcoma and soft tissue sarcoma; NHL, non-Hodgkin's lymphoma; PCU, palliative care unit.

95\% CI: 61.8\%-90.2\%; $P=0.0164$ ) (Figure 4B). These results indicated that the ratio of patients treated near their own residence tended to decrease in proportion to the disease prevalence. In contrast to results previously described, the ratio of patients with OS/STS referred from hospitals outside Okayama prefecture $(28.6 \%$; 95\% CI: $11.3 \%-52.2 \%)$ was significantly higher than those of patients with NHL $(0.0 \%$; 95\% CI: $0.0 \%-9.49 \%$ ) and those of patients treated in the PCU (0.0\%; 95\% CI: 0.0\%-9.74\%). Furthermore, patients with NHL and those who had been treated in our PCU did not reside outside Okayama prefecture (Figure 4C).

\section{Discussion}

A standard treatment guideline for rare cancers is yet to be established due to lack of medical evidence, and treatment modalities vary among medical facilities. Furthermore, because treatment options for recurrent rare cancers are often ineffective, only few patients with rare cancer receive irreproachable treatment in a salvage setting. ${ }^{2,3}$

OS and STS are among the rare cancers with more than 50 histologic subtypes, and treatment outcomes using a multidisciplinary approach have evidently improved over the past few decades in cases of locally advanced disease; however, the clinical outcomes of patients with extended disease or metastatic disease remains poor. ${ }^{7}$ A meta-analysis conducted by Allison et al revealed a significant improvement in the clinical outcome of OS during the 1970s. Combination chemotherapy consisting of anthracycline and alkylating agents for the treatment of advanced or metastatic OS significantly prolonged patients' survival. However, improvements in treatment outcomes have been slow for the past 2 decades because novel, effective drugs have not been developed except olaratumab, which was recently approved in combination with doxorubicin for the treatment of STS by the US Food and Drug Administration. ${ }^{8}$ 
A

Ratio of patients from Okayama city

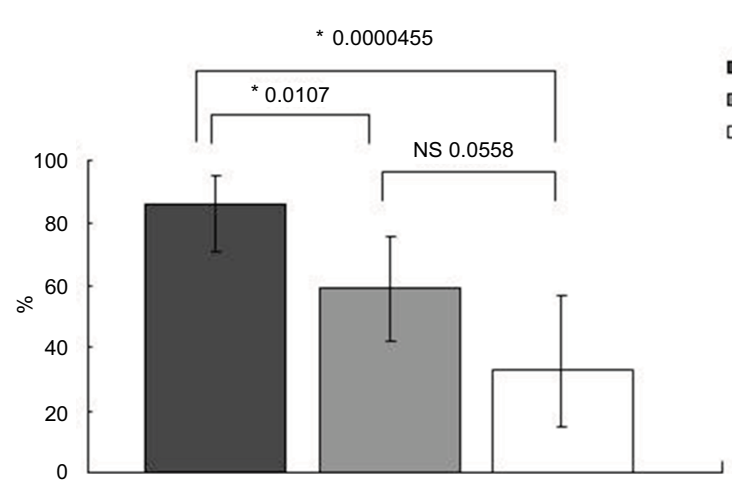

B Ratio of patients from medical

administration area of Okayama prefecture

${ }^{*} 0.0000482$

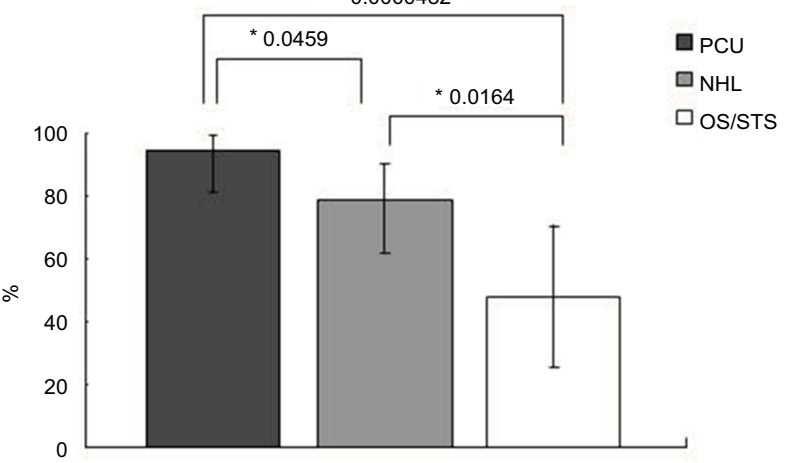

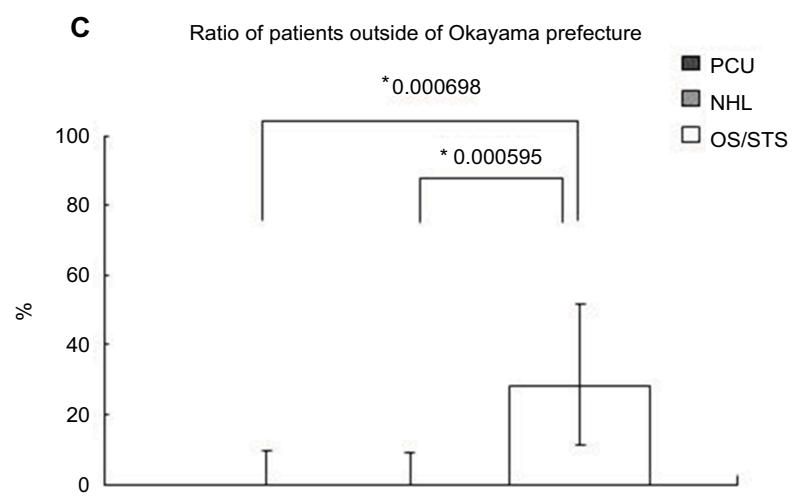

Figure 4 Ratio of patients from Okayama prefecture and those residing outside the prefecture.

Notes: The ratio of patients treated in their own area of residence seemed to decrease in proportion to the disease prevalence (A, B). The ratio of patients with OS/STS who reside outside Okayama prefecture was significantly higher than that of patients with NHL and patients treated in PCU (C). *All P-values corresponded to two-sided tests, and significance was set at $P<0.05$.

Abbreviations: OS/STS, osteosarcoma and soft tissue sarcoma; NHL, non-Hodgkin's lymphoma; PCU, palliative care unit; NS, not significant.

Similar to other rare cancers, conducting clinical trials to establish innovative treatment modalities and developing medical experts are also challenging in OS/STS. Therefore, general physicians in Japanese local cities have difficulty in providing standardized medical care for patients with advanced OS/STS.

OS/STS have specific characteristics, for example, age of onset, primary and metastatic sites, form of metastasis, and specific genes that can induce tumorigenesis. ${ }^{9-11}$ Furthermore, because the prevalence of OS and STS was 0.59 and 3.60 per 100,000 population, respectively, they were thought to be relatively "major" tumors among "rare cancers". ${ }^{4}$ Because of these tumor-specific characteristics, treatment facilities in Japan must be consolidated to establish an effective and innovative therapeutic modality. Moreover, methods for disease control should be considered an urgent problem.

The current status of treatment modalities for patients with OS in Japan was described in the soft tissue tumor registry developed by the Japanese Orthopedic Association in $2010 .{ }^{12}$ In their report, although 168 Japanese patients with
OS were registered in the 55 facilities allover the country, $\sim 70 \%$ of the facilities only attended to less than three patients. These results indicated that Japanese patients with OS/STS have been consulting doctors in other facilities, including small-scale hospitals, and suggested the possibility that the execution of standard treatment throughout the country will be difficult.

To overcome the current status of OS/STS, new attempts in clinical practice are necessary. We, medical and orthopedic oncologists in two different facilities in Okayama city, have been collaborating to provide standard treatments for patients with advanced OS/STS since 2011. The results of our retrospective analysis of OS/STS patients who received combination chemotherapy or palliative treatment from April 2011 to January 2017 were described here. We found that completing medical care within the patient's own medical administration area was difficult because the medical area of patients with OS/STS in local Japanese cities has not been consolidated. Proper treatments which these patients need cannot always be provided unless they find dedicated facilities. Patients 
tend to be desperate, searching for advice and care, and are called "cancer refugees". Thus, some patients with OS/STS might become "cancer refugees" who are unable to receive standard therapy in their area of residence.

Chemotherapy for patients with OS/STS is more toxic than for other solid tumors in general. For example, the frequency of febrile neutropenia is extremely high. ${ }^{13}$ Therefore, the adequate support by clinical oncologists who specialize in cancer chemotherapy is required. Recently, establishment of specialized facilities for orthopedic malignancies, in which a multidisciplinary approach could easily be provided, has been generated allover the world in order to achieve better clinical outcome. Actually, a few facilities have already been founded in each country. ${ }^{14}$ In such a situation, it seems difficult for patients with OS/STS in a regional city to receive adequate treatment. There are few resources for community medicine in Japan. Thus, the orthopedists who are experts in surgical treatment and clinical oncologists who are experts in systemic chemotherapy should be united between facilities in order to satisfy the patients with OS/STS in Japanese regional cities. We believe that this approach might solve the current problems of rare cancers such as OS/STS. This study had a number of limitations. Okayama city is the capital city of Okayama prefecture in Japan. As of April 2017, the city has an estimated population of 721,097 (http://www.city. okayama.jp/soumu/bunsho/bunsho 00162.html). We are not sure whether Okayama city is a representative local city in Japan. Furthermore, the analyses were based on a small sample size and the retrospective study might have a selection bias. Thus, the results should be confirmed in large-scale studies to ensure the generalizability of our data. Finally, the relationship between disease prevalence and ratio of patients who could be treated in their own areas of residence should be analyzed regarding various tumors.

\section{Conclusion}

To the best of our knowledge, the relationship between disease rarity of malignant tumors and the difficulty of treatment completion in the patient's area of residence has not been described. We believe that our report has considerable importance as the findings represent the first step to improve the current treatment status of patients with OS/STS in Japanese regional cities.

\section{Acknowledgments}

We thank Dr Joe Hasei and Dr Tomohiro Fujiwara of the Department of Orthopedics, Okayama University Graduate School of Medicine, for their helpful comments and discussion during the drafting of this study.

\section{Author contributions}

Hiromichi Yamane and Toshiyuki Kunisada planned this study. Toshifumi Ozaki, Nobuaki Ochi, Yoshihiro Honda, Yasunari Nagasaki, Nozomu Nakagawa, Tomoko Yamagishi, and Hidekazu Nakanishi were involved in patient treatment. Nobuaki Ochi, Hiromichi Yamane, and Nagio Takigawa analyzed the data. Hiromichi Yamane and Nagio Takigawa wrote the paper. All authors contributed toward data analysis, drafting and revising the paper and agree to be accountable for all aspects of the work.

\section{Disclosure}

The authors report no conflicts of interest in this work.

\section{References}

1. Gatta G, van der Zwan JM, Casali PG, et al. Rare cancers are not so rare: the rare cancer burden in Europe. Eur J Cancer. 2011;47(17):2493-2511.

2. Japanese Ministry of Health, Labour and Welfare. Study meeting report about the way of the rare cancer care and support. Japanese Ministry of Health, Labour and Welfare; 2015. Available from: http:// www.mhlw.go.jp/file/05-Shingikai-10901000-Kenkoukyoku-Soumuka/0000095429.pdf. Accessed January 24, 2018.

3. Greenlee RT, Goodman MT, Lynch CF, Platz CE, Havener LA, Howe HL. The occurrence of rare cancers in U.S. adults, 1995-2004. Public Health Rep. 2010;125(1):28-43.

4. ganjoho.jp [homepage on the Internet]. Cancer Statistics in Japan 2013. Tokyo: Foundation for Promotion of Cancer Research [updated December 5, 2013]. Available from: http://ganjoho.jp/en/professional/ statistics/brochure/2013_en.html. Accessed January 10, 2018.

5. Fletcher CD, Bridge JA, Hogendoorn PC, Mertens F, editors. World Health Organization Classification of Tumours of Soft Tissue and Bone, 4th ed. Lyon: IARC Press; 2013.

6. Escobar Pinzon LC, Claus M, Zepf KI, Letzel S, Fischbeck S, Weber M. Preference for place of death in Germany. J Palliat Med. 2011;(10): 1097-1103.

7. Kawai A, Yonemori K, Takahashi S, Araki N, Ueda T. Systemic therapy for soft tissue sarcoma: proposal for the optimal use of pazopanib, trabectedin, and eribulin. Adv Ther. 2017;(34):1556-1571.

8. Allison DC, Carney SC, Ahlmann ER, et al. A meta-analysis of osteosarcoma outcomes in the modern medical era. Sarcoma. 2012;2012: 704872 .

9. Kane JM, Finley JW, Driscoll D, Kraybill WG, Gibbs JF. The treatment and outcome of patients with soft tissue sarcomas and synchronous metastases. Sarcoma. 2002;(2):69-73.

10. Penel N, Grosjean J, Robin YM, Vanseymortier L, Clisant S, Adenis A. Frequency of certain established risk factors in soft tissue sarcomas in adults: a prospective descriptive study of 658 cases. Sarcoma. 2008;2008:459386.

11. Hisada M, Garber JE, Fung CY, Fraumeni JF Jr, Li FP. Multiple primary cancers in families with Li-Fraumeni syndrome. J Natl Cancer Inst. 1998;90(8):606-611.

12. Japanese Orthopedic Association Committee on Musculoskeletal Tumor. Soft tissue tumor registry in Japan 2010. Tokyo: National Cancer Center; 2010

13. Bui BN, Chevallier B, Chevreau C, et al. Efficacy of lenograstim on hematologic tolerance to MAID chemotherapy in patients with advanced soft tissue sarcoma and consequences on treatment dose-intensity. J Clin Oncol. 1995;13(10):2629-2636.

14. Robbins L, Shapiro LA, Spielmann MP, Baldi G. CEOs from Orthopaedic Centers Worldwide Meet to Discuss Common Challenges: 2010 Annual Meeting of the International Society of Orthopaedic Centers. HSS J. 2011;7(2):179-182. 
Cancer Management and Research

\section{Publish your work in this journal}

Cancer Management and Research is an international, peer-reviewed open access journal focusing on cancer research and the optimal use of preventative and integrated treatment interventions to achieve improved outcomes, enhanced survival and quality of life for the cancer patient

The manuscript management system is completely online and includes

Submit your manuscript here: https://www.dovepress.com/cancer-management-and-research-journal

a very quick and fair peer-review system, which is all easy to use. Visit $\mathrm{http}: / / \mathrm{www}$.dovepress.com/testimonials.php to read real quotes from published authors. 